\title{
Objective Quantification of Perfusion-Weighted Computed Tomography in the Setting of Acute Aneurysmal Subarachnoid Hemorrhage
}

\author{
Celina Imielinska ${ }^{a, b},^{*}$, Xin Liu $^{a}$, Michael E. Sughrue ${ }^{c}$, Sean Kelly ${ }^{a}$, \\ Eugene Hagiwara ${ }^{\mathrm{d}}$, E. Sander Connolly ${ }^{\mathrm{c}}$, Jr., Anthony L. D'Ambrosio ${ }^{\mathrm{c}}$ \\ ${ }^{a}$ Department. of Biomedical Informatics, Columbia University, New York, NY, USA \\ ${ }^{b}$ Department of Computer Science, Columbia University, New York, NY, USA \\ ${ }^{c}$ Department of Neurological Surgery, Columbia University, New York, NY, USA \\ ${ }^{d}$ Department of Neuroradiology, Columbia University, New York, NY, USA
}

\begin{abstract}
Perfusion-Weighted Computed Tomography (CTP) is a relatively recent innovation that estimates a value for cerebral blood flow (CBF) using a series of axial head CT images which tracks the time course of signal from an administered bolus of intravenous contrast. We introduce a novel computer-based method for objective quantification of CBF values calculated from CTP images. Our method corrects for he inherent variability of the CTP methodology seen in the subarachnoid hemorrhage (SAH) patient population to potentially aid in the diagnosis of cerebral vasospasm (CVS). This method analyzes and quantifies side-to-side asymmetry of CBF and represents relative differences in a construct termed a Relative Difference Map (RDM). Herein, we present our preliminary results that show that analysis of histograms of the RDM in left and right hemispheres, as well as different vascular territories of the brain, can be used for detection and diagnosis of cerebral vasospasm in patients with SAH. While this method has been designed specifically to analyze post-processed CTP images, it could be potentially applied to quantification and analysis of MR perfusion data, as well
\end{abstract}

Keywords: CT; Brain; Perfusion; Cerebral blood flow; aneurysmal subarachnoid; ischemic stroke

* Corresponding author. E-mail address: ci42@ columbia.edu 


\section{Introduction}

Stroke is the third leading cause of death and disability in contemporary society. Subarachnoid hemorrhage (SAH) accounts for approximately 6 to $8 \%$ of all strokes and 22 to $25 \%$ of cerebrovascular deaths. Between 1 million and 12 million people in the United States harbor intracranial aneurysms, and the annual prevalence of aneurysmal SAH (aSAH) in this country is believed to be in excess of 30,000 persons. Despite considerable advances in the diagnosis and treatment of SAH, the outcome remains poor $[1,2]$. The presence of subarachnoid blood is sufficient to produce severe spasm of cerebral arteries. This process is called cerebral vasospasm (CVS). However, the primary mechanism for vasospasm is unknown, and therefore, any patient with aSAH is considered to be at risk for developing CVS [3,4]. Rapid diagnosis and treatment of CVS poses a true diagnostic dilemma and the lack of reliably predictive test often leads to delayed intervention and irreversible neurological injury [5]. Developing a noninvasive, reliable, and sensitive test that could risk stratify patients for CVS during the first days of their hospital admission into high, medium, and low risk populations would be of immense benefit for patients and could potentially decrease health care costs.

Perfusion-Weighted Computed Tomography (CTP) is a relatively recent innovation that utilizes a series of axial head CT images to track the time course of signal from an administered bolus of intravenous contrast [6-9]. These images are then processed using either deconvolution or maximum slope algorithms to extrapolate a numerical value for cerebral blood flow (CBF) [9]. While "bolus tracking" methods may provide accurate quantification of $\mathrm{CBF}$ under controlled conditions, variability in cardiac function, systemic blood pressure, and cerebrovascular tone often seen in the setting of acute SAH makes quantitative and qualitative assessment of these studies both difficult and potentially hazardous [7-9].

While CTP has found some utility in the diagnosis and management of ischemic stroke, its potential use in the diagnosis and management of delayed CVS has not been investigated. Furthermore, since this imaging study is both fast and non- invasive, it is an ideal diagnostic test for this often unstable patient population. Unfortunately, due to the inherent variability described above, there is no currently accepted, standardized method of interpreting these scans. Most commonly, scans are interpreted using the qualitative detection of gross side-to-side asymmetry of CBF, an approach that lends itself to misdiagnosis and potential failure to treat CVS. Recent work with CTP has focused on the development of methods to quantitatively analyze CTP images. Most of these approaches utilize the region of interest (ROI) method [10-13]. In this approach, the clinician circles an ROI on the post-processed CTP image, and the mean CBF is compared to that of the corresponding ROI in he contralateral hemisphere to detect asymmetry. A growing body of data supports improved safety and efficacy of this approach in the setting of acute ischemic stroke, though data for SAH is less complete.

We have developed a novel algorithm for analyzing post-processed CTP images obtained from a standard perfusion CT software package (Siemens Medical Solutions). This method converts CBF values, which must be viewed as meaningless outside of the 
context of a given scan, to relative "difference," which represents side-to-side asymmetry and is a meaningful value in and of itself. This conversion is performed by comparing a small region of the scan to the corresponding region in the contralateral hemisphere, quantifying the degree of relative difference, and representing this quantity of relative difference in $2 \mathrm{D}$ and $3 \mathrm{D}$ in a construct we termed a Relative Difference Map (RDM). We analyze and quantify the amount of "relative difference" in both brain hemispheres and the 6 major vascular territories to assess the degree of hypoperfusion in the regions. The method is automated and has a potential of providing a better and more stable analysis of the perfusion parameters of patients with SAH.

\section{Methods}

Patients presenting to New York Presbyterian Hospital following SAH resulting from the rupture of an angiographically confirmed intracranial aneurysm were enrolled in an IRB approved project in which extensive clinical and long term functional outcome data were collected. DICOM images from the patients were processed by a neuroradiologist, and selected for this analysis.

A simplified model of the human brain can be defined as a symmetric object if corresponding regions of both hemispheres have comparable structural similarity and CBF equivalence. For our analysis, we made the following assumptions based on an accepted human brain anatomy and physiology: (1) In normal cases, the axial CT images of the left and right hemispheres are structurally symmetric and comparable, and there should be no significant relative blood flow difference between the two hemispheres. (2) In abnormal cases, the left and right hemispheres are still structurally symmetric and comparable; but there is significant relative blood flow difference between the two hemispheres, that can be detected using CTP images.

Based on these assumptions, in our preliminary work, we excluded patients with significant midline shift from focal hematoma, hydrocephalus, or focal cerebral edema. In the future, the algorithm should have the capability to automatically identify midline in a curvilinear fashion to correct for this structural asymmetry.

\subsection{Relative Difference Map (RDM)}

Original color DICOM images, Fig.1 (a), are transformed into 8 bit binary format, where the color scales in the original images are normalized into the scale ranging from 0 to 255 Fig.1 (b). Using our stated assumptions, a midline is estimated as a straight line drawn along the anterior-posterior axis through the septum pelucidum to equally divide the brain into two symmetric hemispheres. For quantification of symmetry, the Kolmogorov-Smirnov test [14] is applied to find the greatest statistical discrepancy between the observed and expected cumulative frequencies between two populations, using symmetric 8 by 8 windows in respective brain hemispheres. The average intensities of pixels in the window from one hemisphere are subtracted from those of the contralateral hemisphere, and the absolute difference is divided by the intensity value on the side where CBF reading is relatively larger ("relatively normal hemisphere"). The result is displayed on the side where the reading of the mirrored window is smaller ("relatively abnormal hemisphere") to display the score for relative difference map. 
Fig.1 (c). We can apply the same method to quantify the cerebral blood volume (CBV), and time to peak (TTP) parameters.

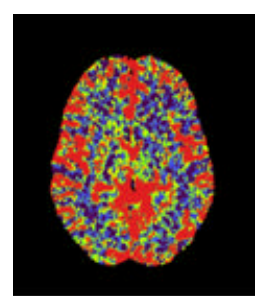

(a)

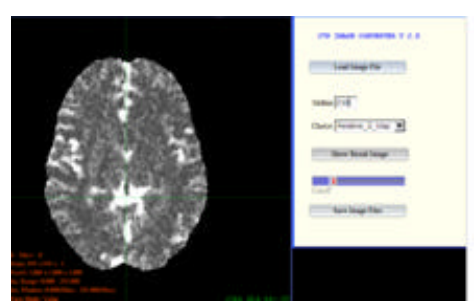

(b)

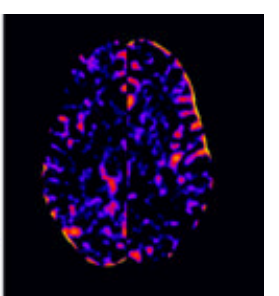

(c)

Fig.1. Relative Difference Map for CBF: (a) CBF image displayed by Siemens' perfusion software, (b) CT input $\mathrm{CBF}$ with selected midline, (c) output RDM.

The resulting RDM is then subdivided into the 6 major cerebrovascular territories, and regional histograms are calculated for each territory. Fig.2 represents a hypothetical distribution of histograms of regions in the RDMs computed for CBF. These curves represent the conceptual basis behind our algorithm. We hypothesize that in disease states, "difference" will cluster in affected territories, and will skew the regional histogram to the right in "problem areas."

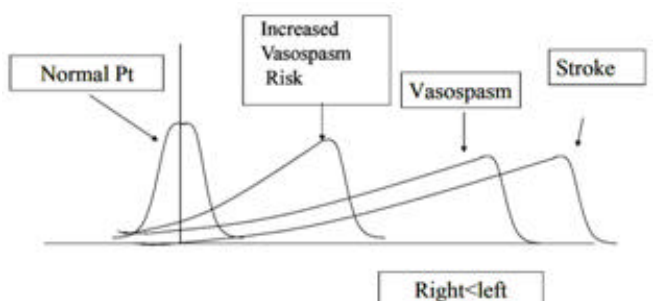

Fig. 2. Hypothesized regional histograms quantified from relative difference maps (RDM) of normal pat ients; and patients with increased vasospasm risk; angiographic vasospasm; and ischemic stroke.

\section{Results}

We present our preliminary results in 3 illustrative cases described below. The images are presented as RDMs processed from CBF maps. Data for patient 2 were kindly provided by Dr. Tomandl, Dept. of Neuroradiology, University of Erlangen-Nuremberg. Patient 1: Patient is a 43 year old man who presented with Hunt and Hess grade $2 \mathrm{SAH}$. Cerebral angiography disclosed a large $(2 \mathrm{~cm})$ MCA aneurysm, which involved the lenticulostriates. The patient's clinical course was unremarkable from a neurological standpoint, with no episodes of CVS detected by daily Transcranial Doppler sonography (TCD), cerebral angiography, or routine neurological examination. On SAH Day 5, the patient underwent CTP depicted in Fig. 3. Regional histograms of vascular territories demonstrate relatively minimal deviation of the curve from zero in all territories, indicating relatively normal levels of perfusion throughout the brain. 
Patient 2: Patient is a 77 year old woman who presented with symptoms consistent with left MCA infarction. Fig. 4 clearly demonstrates large wedge shaped region of severe hypoperfusion in the MCA territory consistent with acute proximal MCA occlusion. When this image has been processed using our algorithm, a clear peak on the far right is seen in the histogram representing the eft MCA territory that is consistent with our theoretical stroke curve as shown in Fig.2. The histograms for other vascular territories have significantly smaller means, and many appear "normal."

Patient 3: Patient is a 36 year old woman who presented with Hunt and Hess grade 4 SAH. Cerebral angiography disclosed a $4 \mathrm{~mm} \times 3 \mathrm{~mm}$ right anterior choroidal artery aneurysm. Her neurological examination improved significantly postoperatively. However, on SAH day 5, she experienced an acute decline in mental status, however neurological exam demonstrated no focal neurological deficit. A CTP performed at that time, Fig.5, was read as normal by an attending neuroradiologist. The patient subsequently developed bilateral arm weakness and was taken for angiography, which revealed severe vasospasm of the right and left MCA and right ACA. This spasm was treated with angioplasty, with significant clinical improvement. Unfortunately however follow-up MRI two months later demonstrated old cerebral infarction in the right frontal lobe. The RDM's demonstrate significant regions of side-to-side asymmetry in the Left MCA and Right ACA territories, consistent with the results seen at angiography. The histograms of these regions, while not as striking as those seen for Patient 2, nevertheless demonstrate significant increases in frequency of significantly mismatched pixels (i.e. shift of curve to the right).

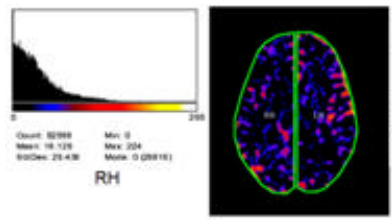

(a)

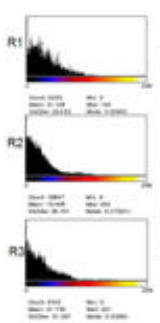

$\equiv=$

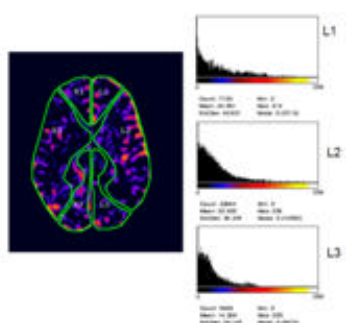

(b)

Fig.3 Patient with "normal" CBF": histograms for (a) Left and Right hemispheres, (b) six vascular territories: L1- Left anterior cerebral artery, L2- Left Middle Cerebral artery, L3 - Left Posterior cerebral artery , R1- Right anterior cerebral artery, R2 - Right Middle Cerebral artery, R3 - Right Posterior cerebral artery.

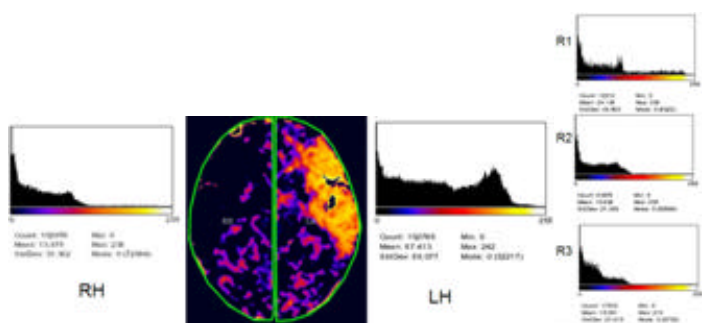

(a)

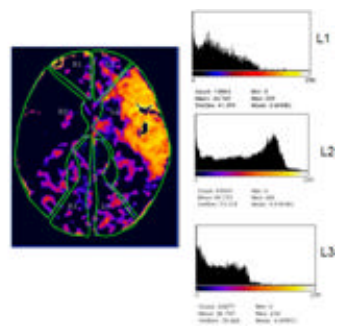

(b)

Fig.4 Patient with is chemic stroke: histograms for (a) Left and Right hemispheres, (b) six vascular territories. 


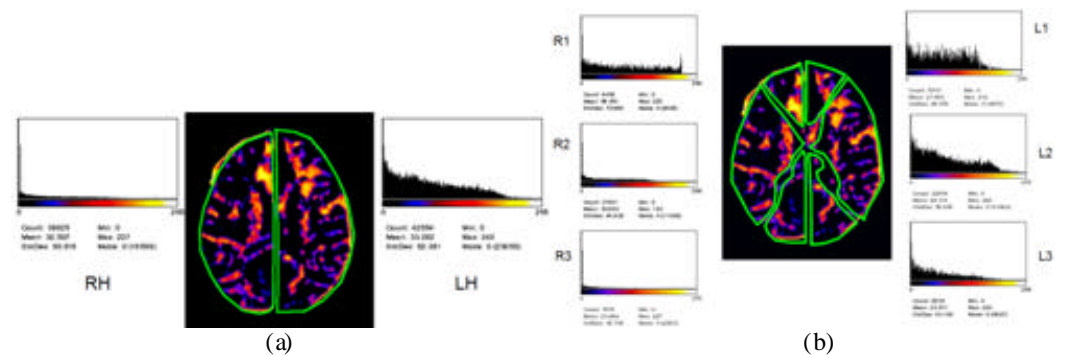

Fig.5 Patient with SAH: histograms for (a) Left and Right hemispheres, (b) six vascular territories.

\section{Conclusions}

While CTP represents a potentially valuable clinically tool in daily practice, rapid, accurate, and safe methods for interpreting the post-processed images are still currently in development. We have begun work on a method of rapidly providing the clinician with an objective, quantification of symmetry of CBF data using relative difference maps. This preliminary study will be followed by thorough analysis of hundreds of patient cases with SAH and acute ischemic stroke to determine the ideal approach to statistical analysis of RDM and its regional histograms. While this method has been designed specifically to analyze post-processed CTP images, it could be potentially applied to quantification and analysis of MR perfusion data, as well.

\section{References}

[1] Ronkainen A, Niskanen M, Rinne J. et al.. Evidence for excess long-term mortality after treated subarachnoid hemorrhage. Stroke. 2001;32:2850-2853

[2] Janjua N, Mayer, S.A. Cerebral vasospasm after subarachnoid hemorrhage Neurocritical Care. 2003;9:113-119

[3] Weir B. The history of cerebral vasospasm. Neurosurgery Clinics of North America. 1990;1:265 -274

[4] Barker FGaHRC. Clinical aspects of vasospasm. Neurosurgery Clinics of North America. 1990;1:277-288.

[5] Sanchez RaP -SJ. Radiologic features of cerebral vasospasm. Neurosurgery Clinics of North America. 1990;1:289-306

[6] Axel L. Cerebral blood flow determination for rapid-sequence computed tomography: theoretical analysis. Radiology 1980;137:679-686.

[7] Roberts HC, Dillon WP, Smith WS. Dynamic ct perfusion to assess the effect of carotid revascularization in chronic cerebral ischemia. Ajnr. 2000;21:421-425

[8] Nabavi DG, Cenic A, Craen RA, et al.. Ct assessment of cerebral perfusion: Experimental validation and initial clinical experience. Radiology. 1999;213:141-149

[9] Ostergaard L, Weisskoff RM, Chesler DA, Gyldensted C, Rosen BR. High resolution measurement of cerebral blood flow using intravascular tracer bolus passages. Part I: Mathematical approach and statistical analysis. MR in Medicine. 1996;36:715-725

[10] Koenig M, Kraus M, Theek C. et al. Quantitative assessment of the ischemic brain by means of perfusion-related parameters derived from perfusion CT. Stroke. 2001;32:431-43

[11] Nabavi DG, Kloska SP, Nam E-M, et al.. Mosaic: Multimodal stroke assessment using computed tomography: Novel diagnostic approach for the prediction of infarction size and clinical outcome Stroke. 2002;33:2819-2826

[12] Klotz. E, König, "perfusion measurements of the brain: using dynamic CT for the quantitative assessment of cerebral ischemia in acute stroke, European Journal of Radiology, 30:170-184, 1999.

[13] Tomandl BF, Klotz E, Handschu R, et al., Comprehensive imaging of ischemic stroke with multisection CT", RadioGraphics, 23:565 -592, 2003.

[14] Chakravarti, Laha, and Roy, Handbook of Methods of Applied Statistics, Vol. I, John Wiley and Sons. 